\title{
Diagnosing Carpal Tunnel Syndrome in Cavanagh Syndrome: A Literature Review and Proposed Electro Physiologic Protocol
}

\author{
Dinesh Kumbhare ${ }^{1 *}$, Najam Mian ${ }^{1}$, Dhanveer Singh ${ }^{2}$, Alasdair TL Rathbone ${ }^{3}$ and Anne Agur ${ }^{4}$
}

${ }^{1}$ Department of Medicine, University of Toronto, Toronto, Ontario, Canada

${ }^{2}$ Royal College of Surgeons in Ireland, Dublin, Ireland

${ }^{3}$ Schulich School of Medicine and Dentistry, University of Western Ontario, London, Ontario, Canada

${ }^{4}$ Department of Surgery, University of Toronto, Toronto, Ontario, Canada

\author{
Abstract \\ Objective: To summarize the literature on the electrophysiology of Cavanagh Syndrome (CS), to present a clinical \\ application and to propose an updated electrophysiologic protocol.
}

Methods: A systematic literature search for CS was carried out and a clinical application is presented.

Results: CS is characterized by congenital thenar hypoplasia and can be associated with other regional muscular and vascular anomalies. The main electrophysiologic abnormality in patients with CS is a reduced amplitude median compound muscle action potential (CMAP) to the thenar eminence, which is also seen in CTS. Radiographic, and electrodiagnostic findings of a patient with co-morbid CS and CTS is presented. Evaluating the median CMAP to the first lumbrical may assist with the diagnosis of CTS in patients with CS.

Conclusions: As CTS and CS can present with thenar atrophy and low amplitude CMAPs, electromyographers should be aware of the clinical and radiographic characteristics of CS and consider electrophysiologic evaluation of the first lumbrical.

Keywords: Electromyography; Electrophysiology; Carpal tunnel syndrome; Cavanagh syndrome; Congenital thenar hypoplasia

Abbreviations: AANEM: American Association of Neuromuscular and Electrodiagnostic Medicine; CMAP: Compound Muscle Action Potential; CS: Cavanagh Syndrome; CTS: Carpal Tunnel Syndrome; EMG: Electromyography; MRI: Magnetic Resonance Imaging; NCS: Nerve Conduction Studies; SNAP: Sensory Nerve Action Potential; US: Ultrasound

\section{Introduction}

This article presents a clinical application of a patient with comorbid Carpal Tunnel Syndrome (CTS) and Cavanagh Syndrome (CS), and discusses how an electromyographer may approach this particular diagnostic workup. CS is an uncommon disorder of the upper extremities which presents with unilateral or bilateral hypoplasia of the Thenar eminence. The clinical, radiological and electrodiagnostic features were first described by Cavanagh et al. [1]. All five patients studied had either unilateral or bilateral underdevelopment of the muscles of the thenar eminence, with normal sensation in the median nerve distribution. In addition, they also reported no response or a weakened response in the muscles of the thumb, when the median nerve at the wrist was stimulated.

A review of available literature did not reveal any estimates or measures of the prevalence of CS. More generally, approximately $11.2 \%$ of all birth deformities of the hand are congenital thumb anomalies, with $4.6 \%$ consisting of thumb hypoplasia/aplasia [2-5]. Over a 19 year period, the overall prevalence of all congenital upper extremity anomalies was reported as 27.2 cases per 10, 000 live births [6]. Due to its rare characteristics, CS is often unrecognized or misdiagnosed. One such misdiagnosis that clinicians tend to make with CS is CTS. CTS is a common idiopathic, nerve entrapment disorder affecting the hand and forearm. Due to combined compression and traction on the median nerve at the wrist, or as a result of inflammation of nearby tendons and tissues, individuals may feel pain, numbness, tingling, paresthesias or weakness in the hand [7].
Electromyographers commonly encounter small amplitude compound muscle action potentials (CMAPs) when recording over the thenar eminence. Most commonly, this is associated with abnormal median sensory nerve studies and subsequently diagnosed as CTS. However, when median sensory potentials are normal and there is no clinical or electrophysiologic evidence of neurogenic thoracic outlet syndrome, cervical radiculopathy, distal myopathy, motor neuron disease or Lambert-Eaton Myaesthenic Syndrome, the electromyographer must consider anomalous innervations and congenital anomalies [8].

Cavanagh Syndrome (CS) is a rare congenital anomaly that clinicians may overlook when investigating etiologies for low amplitude median CMAPs. This article presents an overview of the literature on CS, reports a case of a patient with both CS and CTS and proposes an electrophysiologic protocol that can be applied to patients with CS suspected of having CTS.

\section{Literature Review}

\section{Methods}

A systematic literature search was carried out in the PubMed, CINAHL and Google Scholar databases, until January 2017. Included were case studies, case series, cross-sectional studies and cohort

${ }^{*}$ Corresponding author: Dinesh Kumbhare, Department of Medicine, University of Toronto, Toronto, Ontario, Canada, Tel: 416593422; E-mail: dinesh.kumbhare@uhn.ca

Received June 09, 2017; Accepted June 22, 2017; Published June 28, 2017

Citation: Kumbhare D, Mian N, Singh D, Rathbone ATL, Agur A (2017) Diagnosing Carpal Tunnel Syndrome in Cavanagh Syndrome: A Literature Review and Proposed Electro Physiologic Protocol. J Pain Manage Med 3: 125.

Copyright: (c) 2017 Kumbhare D, et al. This is an open-access article distributed under the terms of the Creative Commons Attribution License, which permits unrestricted use, distribution, and reproduction in any medium, provided the original author and source are credited. 
studies using the search terms "Cavanagh," "Cavanaugh," "Congenital Thenar Hypoplasia" and variants thereof. All cross-references were checked. Exclusion criteria were articles that were not in English, those discussing global developmental syndromes, and those not discussing thenar atrophy $[9,10]$.

\section{Results}

The search terms yielded 89 results (Figure 1). Five additional articles were identified via cross-references. Ninty-four articles were screened of which 69 were excluded due to the exclusion criteria. The remaining 25 articles received full-text review of which 13 articles were excluded as per the exclusion criteria. Twelve case series and reports comprising 25 patients were included in the qualitative synthesis and are summarized.

Based on the lack of high quality evidence, only qualitative analysis of typical characteristics of CS is possible. Seven of the 12 articles specified the individual muscles that were hypoplastic in CS, with an absence of Opponens Pollicis (OP) in $80 \%$ of cases, the Abductor Pollicis Brevis (APB) in $86.6 \%$ of cases, and the Flexor Pollicis Brevis (FPB) in $57.1 \%$ of cases. The 5 remaining articles, which did not specify the individual absent thenar muscles, only reported that there was generalized hypoplasia of the thenar muscles. One hundred percent of all reported cases presented with weakness, all but one case $(95.8 \%)$ had an absence of pain and paresthesias and all but one case (95.8\%) had an absence of neurologic signs [10].

These 2 apparently atypical presentations included (Table 1) an 11-year-old girl with a history of pain on writing and a 23 -year-old man with positive Tinel's and Phalen's signs. Additional findings of

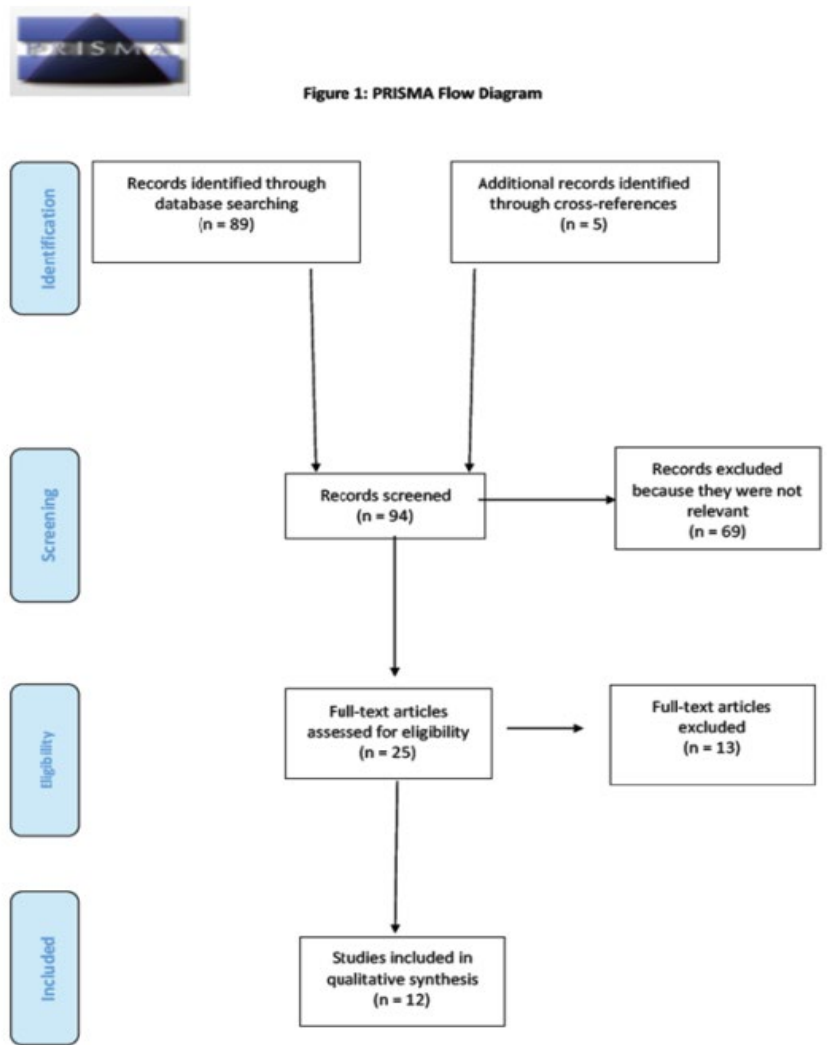

Figure 1: PRISMA flow diagram.
CS included an absent Flexor Pollicis Longus (FPL) in 29\%, ankylosis of the interphalangeal joints in $29 \%$ of cases, thumb anomalies in $25 \%$ of cases, adduction contractures in $16.6 \%$ of cases and radial deviation in $8.3 \%$ of cases.

Seven out of 12 articles reported on radiographic abnormalities. A slender or hypoplastic 1st metacarpal was the most common radiologic finding with a frequency of $33.3 \%$. An absent radial artery or impaired radial circulation, determined through Doppler imaging and Allen's test, was present in $12.5 \%$ of cases.

Electrophysiologic results were reported in 9 of 12 studies (75\%). Three of these articles did not report detailed findings. Two reported only that the median nerve was intact $[11,12]$, and 1 reported only that stimulation of the median nerve did not cause thumb movement.

The articles presenting more detailed electrophysiologic findings reported 10 total patients with decreased or absent median CMAPs in $100 \%$ of cases, and normal median sensory nerve action potentials (SNAPs) in $80 \%$ of cases. There were no reports of abnormal median $\mathrm{F}$ waves or denervation potentials. One series of 5 patients reported volitional activity was absent or reduced in all cases. These reports should be interpreted with caution due to the small sample sizes, incomplete electrophysiologic assessments, and lack of controls [13].

Based on the literature review, CS seems to most commonly present with an absence of APB $(86.6 \%)$ and/or OP $(80 \%)$, weakness (100\%), and a lack of pain, paresthesias and neurologic signs $(95.8 \%)$. Electrophysiologic findings include an absent or reduced CMAP (100\%) and a normal SNAP (80\%). Other findings such as thumb hypoplasia, impaired radial circulation, radial deviation, adduction contractures, ankylosis, and absence of FPL are less likely to be part of the typical presentation. Electrophysiologic findings seem to include decreased or absent median CMAPs and normal SNAPs, although there is a paucity of data from which to draw conclusions [14].

\section{Clinical Application}

A 59-year-old right-handed female presented to the electromyography clinic for assessment of bilateral hand numbness, paresthesias, and pain. She complained of gradually progressive, bilateral paresthesias in both hands involving all 10 digits, with medial radiation into the inferior forearm. Any activities with the hands aggravated her symptoms [15]. She also noted erythema and swelling with activities. Grip strength had subjectively decreased over 3 years. Her past medical history was positive for congenital right thenar atrophy, hypothyroidism, osteopenia, dyslipidemia, shingles, and B12 deficiency. Medications included levothyroxine, naproxen, doxepin, cyclobenzaprine, pregabalin, and colesevalam. Neurological examination revealed reduced right thenar bulk on the right (Figure 2).

There was no other focal atrophy in the hand or the extremities. There were no involuntary movements seen. She had normal tone, $2+$ upper extremity reflexes and negative Hoffman's signs bilaterally. Strength was $5 / 5$ in the major and minor muscles of the upper and lower extremities, with exception of grade $3 / 5$ strength in right thumb abduction. There was no loss of pinprick sensation in the median nerve distribution, no splitting of the 4th finger, and no gradient distribution sensory loss. Proprioception and vibration testing was intact. Tinel's and Phalen's tests were positive on the right and negative on the left [16-19].

\section{Electrophysiologic studies were performed}

The median motor study of the right abductor pollicis brevis had 


\begin{tabular}{|c|c|c|c|c|c|c|c|c|c|c|c|c|c|c|c|}
\hline 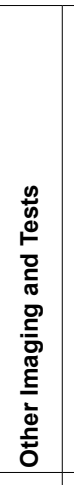 & $\stackrel{0}{z}$ & 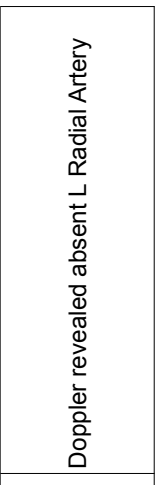 & 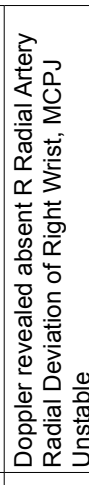 & 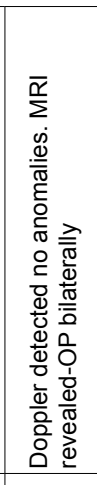 & 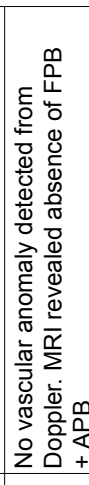 & 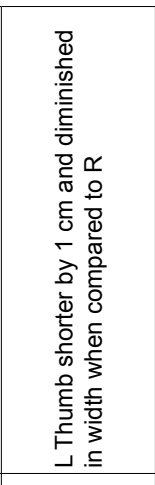 & 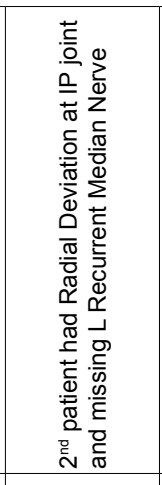 & 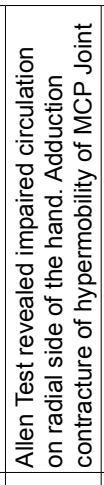 & 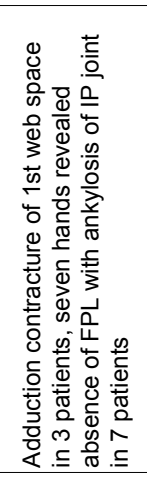 & 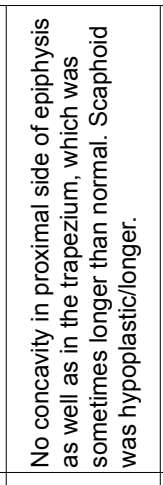 & 足 & 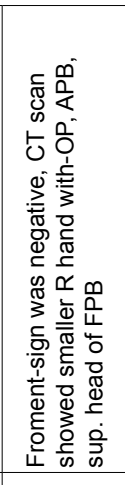 & 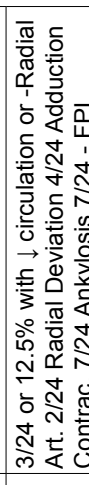 & & 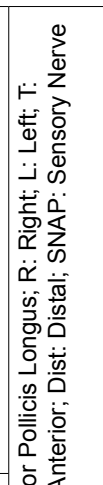 \\
\hline 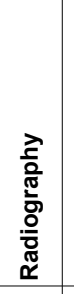 & 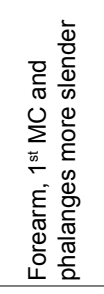 & 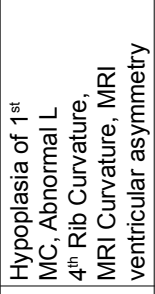 & 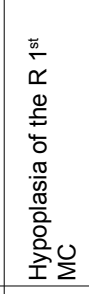 & 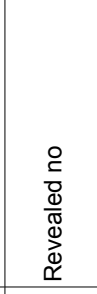 & 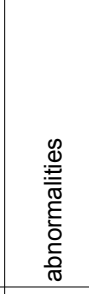 & 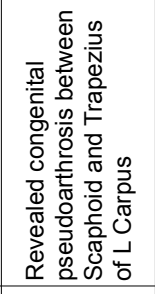 & 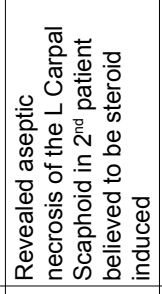 & 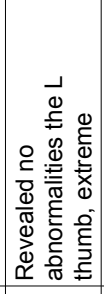 & 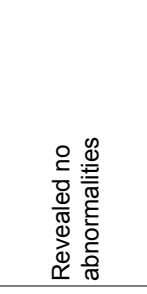 & 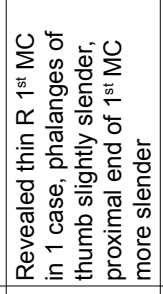 & 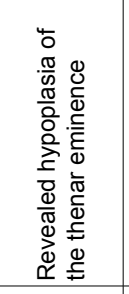 & 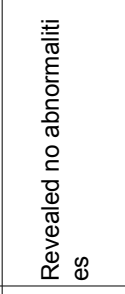 & 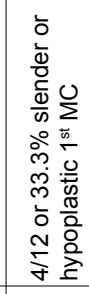 & & 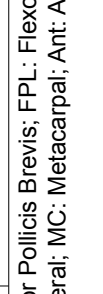 \\
\hline$\frac{0}{z}$ & 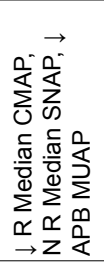 & 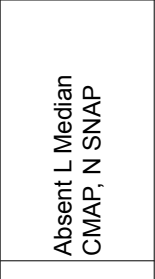 & $\stackrel{\infty}{z}$ & 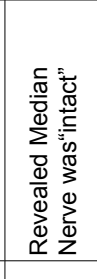 & 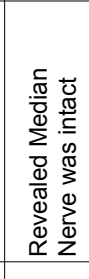 & 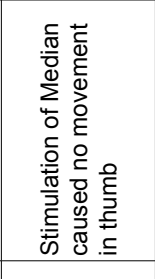 & 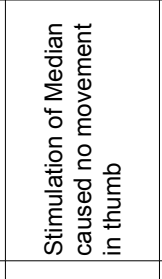 & $\stackrel{0}{z}$ & 0 & 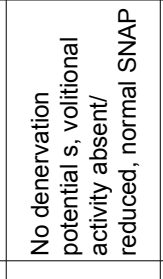 & 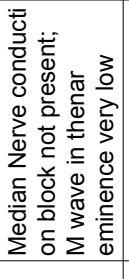 & 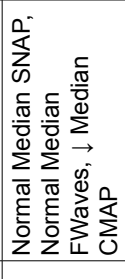 & 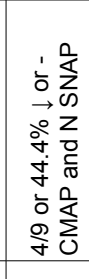 & & 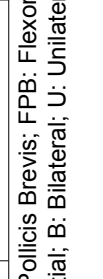 \\
\hline 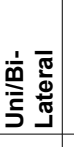 & 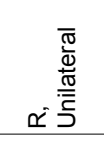 & 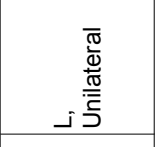 & 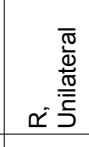 & 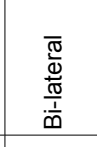 & 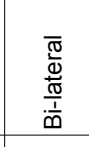 & 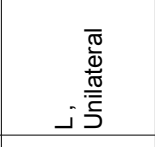 & 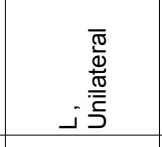 & 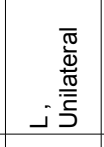 & 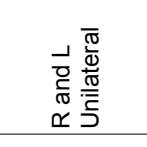 & 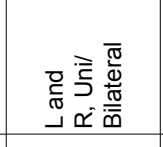 & ¿n & 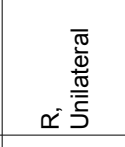 & 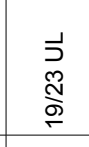 & $\begin{array}{l}\vec{m} \\
\stackrel{N}{\mathscr{V}} \\
\vec{f}\end{array}$ & 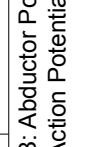 \\
\hline | & $\underline{n}$ & 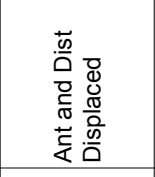 & 悉 & $\stackrel{\infty}{z}$ & $\stackrel{\infty}{z}$ & 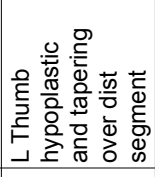 & 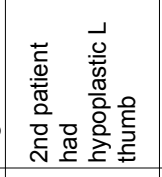 & $\underline{n}$ & 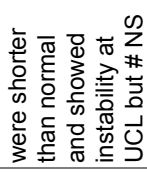 & $z$ & 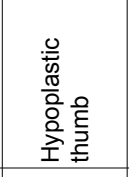 & & 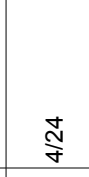 & $\begin{array}{l}\stackrel{\circ}{\circ} \\
\stackrel{\circ}{\circ}\end{array}$ & 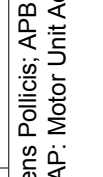 \\
\hline 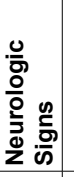 & 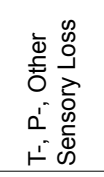 & 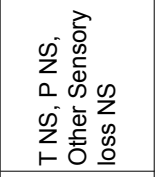 & 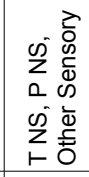 & 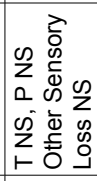 & 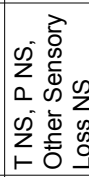 & 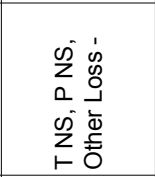 & 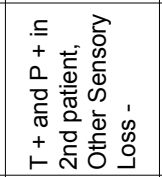 & 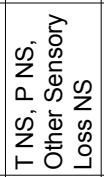 & 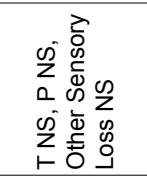 & 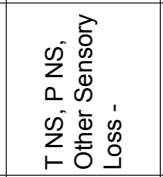 & 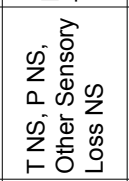 & 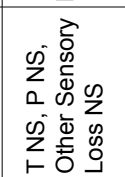 & 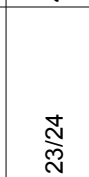 & $\begin{array}{l}\infty \\
\infty \\
\leftrightarrow\end{array}$ & 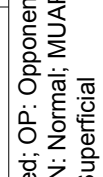 \\
\hline 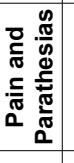 & ' & ' & ' & ' & & & & & & & & & 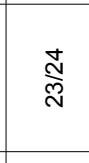 & $\begin{array}{l}\infty \\
\stackrel{\infty}{\infty} \\
\infty\end{array}$ & 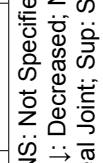 \\
\hline 离品 & + & + & + & + & + & + & + & + & 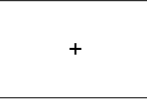 & + & + & + & 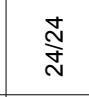 & ○े & 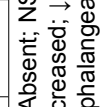 \\
\hline$\frac{m}{\frac{m}{L}}$ & $\stackrel{\text { N }}{z}$ & ñ & z) & + & & & + & & $z$ & $\stackrel{\infty}{z}$ & $\dot{z}$ & & 字 & 号 & 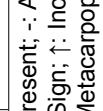 \\
\hline$\frac{m}{\frac{0}{\alpha}}$ & $\stackrel{\text { N }}{z}$ & $\stackrel{\infty}{z}$ & $\stackrel{n}{2}$ & + & 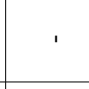 & & $\bar{F}_{F}$ & & & ID & $\bar{z}$ & & 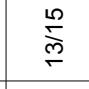 & $\begin{array}{l}\stackrel{0}{0} \\
\dot{0} \\
\infty\end{array}$ & 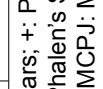 \\
\hline o & ñ & $\underline{\infty}$ & n & ' & + & + & $T^{+}$ & & & D & ñ & & 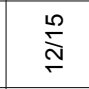 & 今े & 离 \\
\hline $\begin{array}{l}\bar{\Phi} \\
\text { z } \\
\text { z } \\
\end{array}$ & 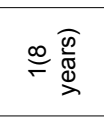 & 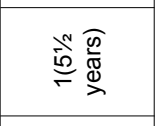 & $\cong \stackrel{\frac{\widehat{D}}{\mathbb{N}}}{\stackrel{N}{\sim}}$ & 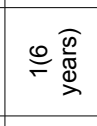 & 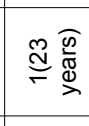 & 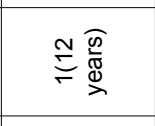 & 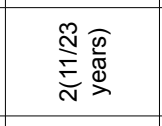 & 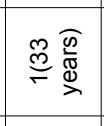 & 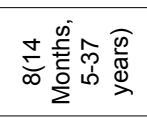 & 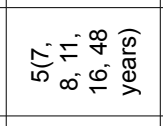 & 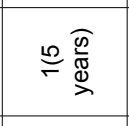 & 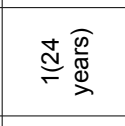 & 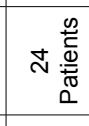 & & 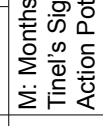 \\
\hline ò & 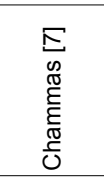 & 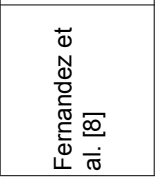 & 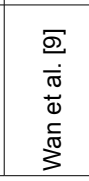 & 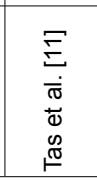 & 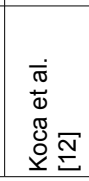 & 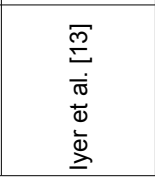 & 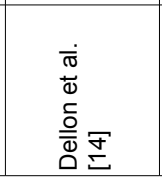 & 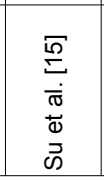 & 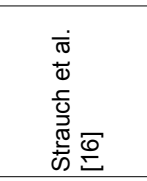 & 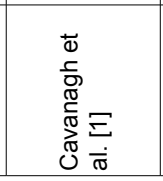 & 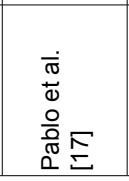 & 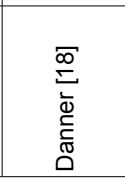 & \# & & \\
\hline
\end{tabular}


Citation: Kumbhare D, Mian N, Singh D, Rathbone ATL, Agur A (2017) Diagnosing Carpal Tunnel Syndrome in Cavanagh Syndrome: A Literature Review and Proposed Electro Physiologic Protocol. J Pain Manage Med 3: 125.

Page 4 of 6

a normal latency and conduction velocity with a reduced amplitude of $1.0 \mathrm{mV}$. (Table 2). Bilateral median motor NCS from the 1st lumbrical showed a $49 \%$ decrease in the right lumbrical amplitude compared to the left $(3.2 \mathrm{mV}$ vs. $6.3 \mathrm{mV})$ although still within normal limits $(<0.7$

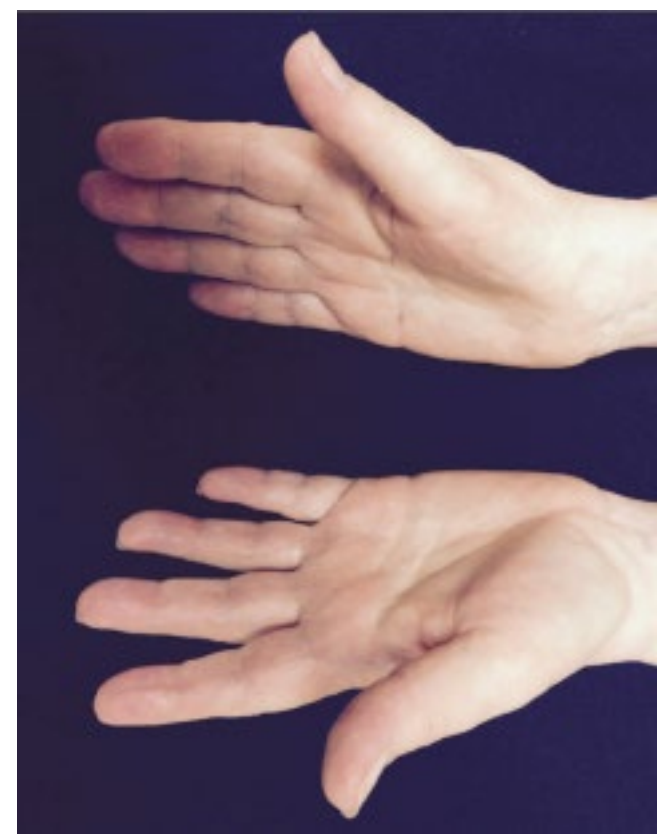

Figure 2: Picture of case showing mild relative right thenar atrophy.
$\mathrm{mV}$ and $<59 \%$ difference; from Buschbacher and Prahlow as no lab normal values are established) [20]. Sensory studies of the median and ulnar nerves were performed in an antidromic manner and were within normative values. The combined sensory index (CSI) [21] was calculated as $0.8 \mathrm{~ms}$, below the value of $1.1 \mathrm{~ms}$ for diagnosis of CTS. Electromyography (EMG) was deferred due to thenar hypoplasia.

Plain radiography of the hands did not reveal any phalangeal hypoplastic changes. Sonographic evaluation of the right forearm measured cross-sectional median nerve area of $8 \mathrm{~mm}^{2}$. Dynamic sonographic evaluation showed a freely moving median nerve within the carpal tunnel. Interestingly, the right radial artery was absent distal

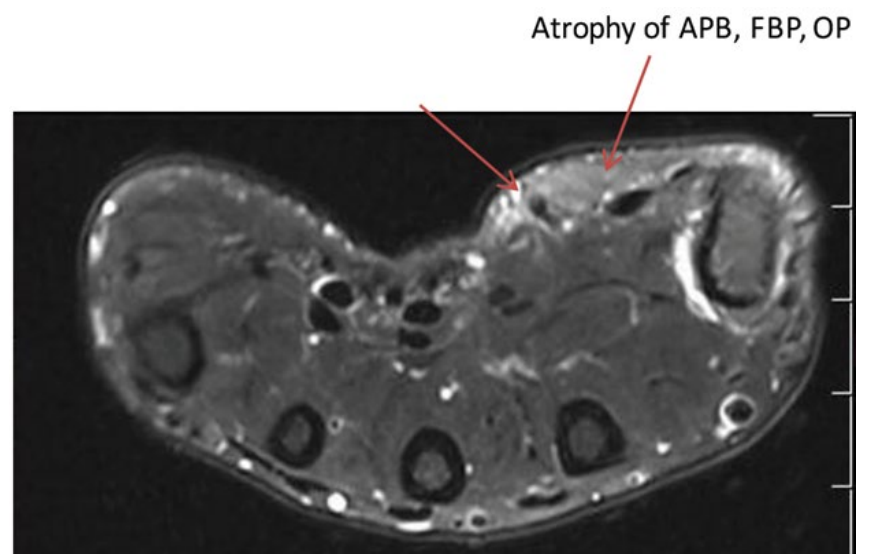

Figure 3: Axial T2 fat-saturated image of the right thenar eminence.

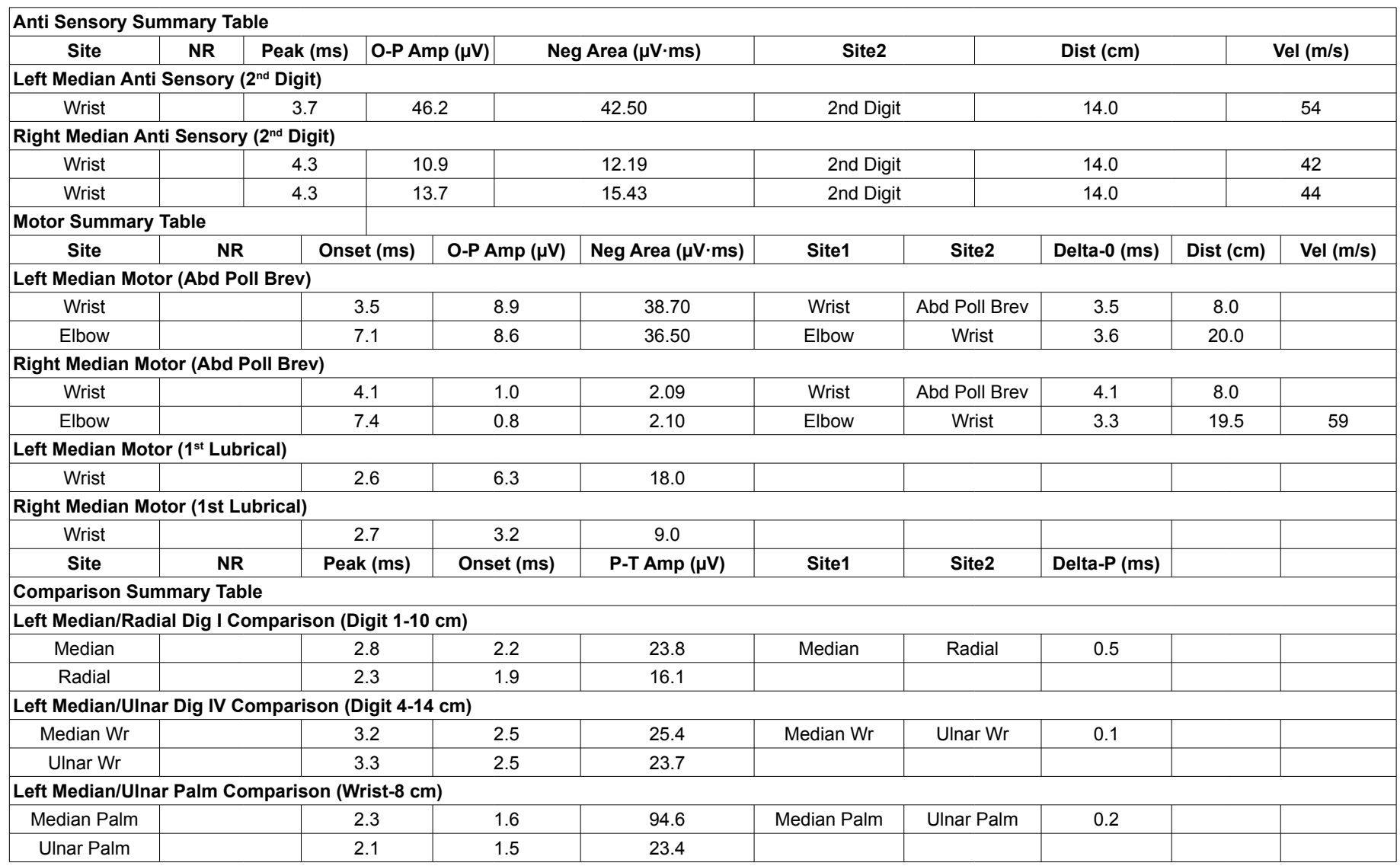

Table 2: Nerve conduction studies. 
to the brachial bifurcation. This was afterwards clinically correlated with a positive right Allen's test. Fatty infiltration and diffuse volume was noted in the right thenar eminence, which made individual assessment of the muscles difficult. An MRI of her right hand (Figure 3) showed marked atrophy of the abductor pollicis brevis, flexor pollicis brevis, mild atrophy of opponens pollicis. The adductor pollicis was normal. There was mild increased signal along the length of the visualized median nerve. The overlying flexor retinaculum was normal. The conclusion was no MRI evidence of carpal tunnel syndrome.

\section{Discussion}

\section{Differential diagnosis of low thenar bulk or low amplitude thenar cmap}

Finding low thenar bulk or low amplitude motor potentials at the thenar eminence is common in clinical practice. Typically, as with most peripheral nerve or muscle findings, the differential diagnosis is first driven by localization. Muscle, neuromuscular junction, peripheral nerve, plexus, root and anterior horn cells are all possible localizations of this finding. Neuromuscular junction disorders, myopathic disorders and anterior horn cell disorders, discussed elsewhere, often have numerous characteristic clinical and electrophysiologic findings outside of the distribution of the thenar eminence. In cases where sensory symptoms and findings are absent; root, plexus, and most peripheral nerve localizations (excluding exclusively motor nerve branches) are unlikely localizations, as typically motor symptoms and electrophysiologic findings do not occur until more severe grades of injury (i.e. after sensory findings are already present) [22]. Peripheral nerve injuries may also show segmental slowing, and radiculopathies are diagnosed in multiple muscles of the same myotome and can include abnormal paraspinal muscle findings and late responses. If there are no clinical or electrophysiologic features of the aforementioned diagnoses, the only remaining possible localizations are anatomic variation in nerve supply or congenital hypoplasia of thenar muscles. Anatomic variation in nerve supply to the thenar eminence is tested through stimulation of the ulnar nerve while recording over the thenar eminence. It is important to stimulate the ulnar nerve in many ascending locations to establish if the fibers supplying the thenar eminence originate in the ulnar nerve (Riche-Cannieu anastomosis) or originate in the median nerve and crossover to the ulnar nerve (MartinGruber anastomosis). CS and other congenital thenar hypoplasias can be identified specifically by a normal first lumbrical despite low amplitudes over the thenar eminence [23].

\section{Diagnosis of cts in patients with cs}

Clinically, thenar hypoplasia or low amplitude CMAPs may be misinterpreted as neurogenic thenar atrophy as found in carpal tunnel syndrome (CTS). However as noted above, patients with typical CS do not have symptoms such as numbness, paresthesias, or nocturnal pain. While both CTS and CS may have low-amplitude CMAPs, electrophysiologic testing in CS thus far has shown normal sensory potentials [24]. In CS, the median sensory supply to the hand is not affected, with the skin over the thenar eminence being supplied by the palmar cutaneous branch, and the volar thumb, index finger, middle finger and lateral half of the ring finger supplied by the digital cutaneous branches of the median nerve $[1,25,26]$. Other differences include normal conduction velocity, normal distal latency, and normal insertional activity in isolated CS, all of which may be abnormal in CTS. If thenar muscles appear normal but low amplitude CMAPs are present over the thenar eminence, anomalous branches of the median nerve or an all-ulnar hand must also be considered [27-36].
Difficulties arise diagnosing CTS in a patient with known or suspected CS. Due to the absence of the thenar muscles (abductor pollicis brevis, flexor pollicis brevis, and opponens pollicis (Table 1) in CS, electrophysiologic testing of the median motor supply to the thenar eminence is not a viable option to detect abnormalities.

\section{Simultaneous diagnoses of cavanagh syndrome and carpal tunnel syndrome}

Clinical and radiographic characteristics suggestive of CS are described in the literature review. The patient in the case report had clinical findings of CS along with MRI findings of marked atrophy of APB and FPB, and mild atrophy of OP. The ultrasound also showed absence of the distal radial artery. However, this patient also had symptoms suggestive of sensory impairment and progressive weakness which suggested a concomitant lesion, proximal to the neuromuscular junction.

The approach to patients with two suspected problems with potentially overlapping signs and symptoms can be difficult. In this case, it is ideal for the physician to first select specific findings that indicate that a congenital disorder is clearly present. For the diagnosis of CS, with or without CTS, we propose this finding is a difference in motor CMAP amplitudes between the thenar eminence and the first lumbrical, as the lumbrical is uninvolved in CS. In our patient, there was a difference in amplitudes between the first lumbrical and the thenar eminence, suggesting CS.

Once this congenital disorder has been identified, the remainder of the assessment should assess for other differential diagnoses. In the case of CS, measuring first lumbrical motor amplitudes in addition to thenar CMAP amplitudes seemed to be helpful as there was a $49 \%$ sideside difference in the first lumbrical CMAPs. The subjective complaints of sensory loss and progressive weakness, along with positive Tinel's and Phalen's signs on examination suggested investigation for CTS. Potential diagnostic investigations for CTS included an ultrasound which showed a normal median nerve cross-sectional area of $8 \mathrm{~mm}$ [37], and an MRI which did not show any evidence of compressive median neuropathy. Thereby, in this patient with CS, electrophysiologic studies seemed to be a complimentary and useful diagnostic tool for the evaluation of co-morbid CTS.

Future high-quality studies are required in order to develop a greater understanding of congenital hypoplasias with concomitant peripheral nervous system disorders. In the interim, based on our findings from the literature review and case study, we propose a protocol for electrophysiologic studies in patients where CS is clinically suspected (adapted from the AANEM protocol for CTS) $[38,39]$.

\section{Conclusion}

Cavanagh Syndrome is characterized by a congenital hypoplasia of thenar muscles. Evaluation of co-morbid carpal tunnel syndrome can potentially be evaluated with an electrophysiologic protocol that includes interpretation of the median motor nerve conduction studies to the first lumbrical.

\section{References}

1. Cavanagh NP, Yates DA, Sutcliffe J (1979) Thenar hypoplasia with associated radiologic abnormalities. Muscle Nerve 3: 431-436.

2. Tay SC, Moran SL, Shin AY, Cooney WP (2006) The hypoplastic thumb. J Am Acad Orthop Surg 14: 354-366.

3. Lister G (1991) Pollex abductus in hypoplastic and duplication of the thumb. J Hand Surg16: 626-633. 
Citation: Kumbhare D, Mian N, Singh D, Rathbone ATL, Agur A (2017) Diagnosing Carpal Tunnel Syndrome in Cavanagh Syndrome: A Literature Review and Proposed Electro Physiologic Protocol. J Pain Manage Med 3: 125.

4. Kozin SH (2003) Upper extremity congenital anomalies. J Bone Joint Surg 85: 1564-1576.

5. Kleinman WB (1990) Management of thumb hypoplasia. Hand Clin 6: 617-641.

6. Goldfarb CA, Shaw N, Steffen JA, Wall LB (2017) The prevalence of congenital hand and upper extremity anomalies based upon the new york congenital malformations registry. J Pediatr Orthop 37: 144-148.

7. Chammas MM (2014) Carpal tunnel syndrome. Chirurgie de la main 33: 75-94.

8. Fernandez D, Gupta S, Fallon P (2009) Congenital thenar hypoplasia with absent radial artery: A case report. Eur J Paediatr Neurol 13: 277-279.

9. Wan K, Pang I (2014) Congenital thenar hypoplasia with absent radial artery: A case report. J Ortho Trauma Rehab

10. Kuntz NL (1997) Pediatric clinical electromyography.

11. Taş S, Top H (2015) Bilateral congenital absence of the opponens pollicis muscle-a case report. Hand 10: 143-146.

12. Koca K, Ekinci S, Ege T, Ozyurek S, Kurklu M, et al (2012) Bilateral congenita absence of flexor pollicis brevis and abductor pollicis brevis muscles with bilateral thenar atrophy-a case report. Clin Med Insight Arthritis Musculoskelet Disord 5: 59-62.

13. Iyer KM, Stanley JK (1982) Congenital absence of flexor pollicis brevis and abductor pollicis brevis. Hand; 14: 313-316.

14. Dellon AL, Rayan G (1981) Congenital absence of the thenar muscles-report of two cases. J Bone Joint Surg 63: 1014-1015 .

15. SU CT , Hoopes JE, Daniel R (1972) Congenital absence of the thenar muscles innervated by the median nerve-report of a case. J Bone Joint Surg Am 54: 1087-1090.

16. Strauch B, Spinner M (1976) Congenital anomaly of the thumb-absent intrinsics and flexor pollicis longus. J Bone Joint Surg Am 58:115-118.

17. Pablo MJ, Costa M, Carvajal P, Saenz De Cabezon A, et al (2009) Cavanagh syndrome in a 5-year-old boy-differential diagnosis with carpal tunnel syndrome. Clin Neurophysiology.

18. Danner R (1983) Unilateral thenar hypoplasia. Clin Neurol Neurosurg 85: 123-128.

19. Buschbacher RM, Prahlow N (2006) Manual of nerve conduction studies. Demos Medical Publishing, New York, USA.

20. Bland, JD (2000) A neurophysiological grading scale for carpal tunne syndrome. Muscle Nerve 23: 1280-1283.

21. Preston D, Shapiro B (2003) Electromyography and neuromuscular disorders. Clinical- Electrophysiological Correlations Mcgill J Med 3: 269-273.

22. Lee KH, Hwang CM, Kim MS, Kim HS, Ahn KH (1998) A case report of congenital carpal tunnel syndrome. J Korean Acad Rehabil Med 23: 752-755.

23. Sachs GM, Raynor EM, Shefner JM (1995) The all ulnar motor hand without forearm anastomosis. Muscle Nerve 18: 309-313.
24. Kimura J, Murphy MJ, Varda DJ (1976) Electrophysiological study of anomalous innervation of intrinsic hand muscles. Arch Neurol 33: 842-844.

25. Kuvat SV, Özçakar L, Yazar M (2010) A foregoing thenar muscular branch of the median nerve. Indian J Plast Surg 43: 106-107.

26. Lanz U (1977) Anatomical variations of the median nerve in the carpal tunnel. J Hand Surg Am 3: 44-53.

27. Siverhus SW, Kremchek TE, Smith WR, Basch TM, Drake RL (1989) A cadaveric study of the anatomic variations of the recurrent motor branch of the median nerve. Orthop Rev 18: 315-320.

28. Linburg RM, Albright JA (1970) An anomalous branch of the median nerve-a case report. J Bone Joint Surg Am 53: 182-183.

29. Rockwell WB, Stone B, Zakhireh M (2001) Three thenar motor branches of the median nerve. Ann Plast Surg 46: 661-662.

30. Graham WP (1973) Third variations of the motor branch of the median nerve at the wrist-a case report. Plast Reconstr Surg 51: 90-92.

31. Eiken O, Carstam N, Eddeland A (1971) Anomalous distal branching of the median nerve - case reports. Scand J Plast Reconstr Surg 5: 149-152.

32. Kessler I (1969) Unusual distribution of the median nerve at the wrist - a case report. Clin Orthop Relat Res 67: 124-126.

33. Robinson LR, Micklesen PJ, Wang L (1998) Strategies for analyzing nerve conduction data - superiority of a summary index over single tests. Muscle Nerve 21:1166-1171.

34. Gazioglu S, Boz C, Cakmak V (2011) A electrodiagnosis of carpal tunne syndrome in patients with diabetic polyneuropathy. Clin Neurophysio 123:1463-1469.

35. Katz JN, Stirrat C, Larson MG, Fossel AH, Eaton HM, et al (1990) A selfadministered hand symptom diagram in the diagnosis and epidemiologic study of carpal tunnel syndrome. J Rheumatol 17:1495-1498.

36. Ziswiler HR, Reichenbach S, Vögelin E, Bachmann LM, Villiger PM et al(2005) Diagnostic value of sonography in patients with suspected carpal tunne syndrome-a prospective study. Arthritis Rheum 53: 304-311.

37. Cartwright MS, Hobson-Webb LD, Boon AJ, Alter KE, Hunt $\mathrm{CH}$, Flores VH Werner RA, Shook SJ, Thomas TD, Primack SJ, Walker FO (2012) American Association of Neuromuscular and Electrodiagnostic Medicine. Evidencebased guideline-neuromuscular ultrasound for the diagnosis of carpal tunne syndrome. MuscleNerve 46: 287-293.

38. Foley BS, Buschbacher RM (2006) Establishing normal nerve conduction values- Lumbrical and Interosseous responses. Journal of Long-Term Effects of Medical Implants.

39. Fitz WR, Mysiw WJ, Johnson EW( 1990) First lumbrical latency and amplitudecontrol values and findings in carpal tunnel syndrome. Am J Phys Med Rehabil 69:198-201. 